\title{
Extracting Domain Information using Deep Learning
}

\author{
Amit Gupta \\ agupta@tacc.utexas.edu \\ Texas Advanced Computing Center, \\ University of Texas at Austin \\ Austin, Texas, USA
}

\author{
Weijia Xu \\ xwj@tacc.utexas.edu \\ Texas Advanced Computing Center, \\ University of Texas at Austin \\ Austin, Texas, USA
}

\author{
Pankaj Jaiswal \\ Department of Botany and Plant \\ Pathology, Oregon State University \\ Corvallis, Oregon, USA \\ jaiswalp@science.oregonstate.edu
}

\author{
Crispin Taylor \\ American Society of Plant Biologists \\ Rockville, Maryland, USA \\ ctaylor@aspb.org
}

\author{
Jennifer Regala \\ American Society of Plant Biologists \\ Rockville, Maryland, USA \\ jregala@aspb.org
}

\begin{abstract}
Across various scientific domains, digital publication of technical documents, often in the form of conference/journal article submissions, are the first accessible instance of new human knowledge in these respective fields. Synthesizing and curating this information is a slow and difficult process and often requires non-trivial human expertise. Given the ever increasing rate of these publications and the natural limitations of manual approaches, a computational solution to this problem is the paramount need of the hour. One of the central tasks is to extract important phrases and terminologies from scientific article. Although many tools are available to extracting keywords and named entities from a document, a key challenge is to determine how important they are with regard the context of the entire document. In some cases, important entities to an article might be a new vocabulary that haven't, or rarely, appeared from previous data. In other cases, there are also many other existing entities that are less important for the particular article but weighted more significantly from models based on prior knowledge. In this paper, we investigate how deep learning methods may be used to address this issue.
\end{abstract}

We have developed a computational tool to provide entity extraction and expert curation functionality. The tool has been integrated with the publication pipeline used in American Society of Plant Biologists. Using the author feedback mechanism in our deployed tool we were able to create a expert user annotated dataset based on articles submitted over an entire year. Using this new gold standard dataset for supervised training, we are now able to contrast several methods for the entity extraction task. We use the NeuroNER tool to investigate the effectiveness of deep neural network in this task and also contrast it with other tools using a variety of different methods such as ABNER (using CRF) and DIVE (using an ensemble of regular expression rules, keyword dictionaries and ontology files). Our results show that DIVE ensemble of methods have higher

Permission to make digital or hard copies of all or part of this work for personal or classroom use is granted without fee provided that copies are not made or distributed for profit or commercial advantage and that copies bear this notice and the full citation on the first page. Copyrights for components of this work owned by others than the author(s) must be honored. Abstracting with credit is permitted. To copy otherwise, or republish, to post on servers or to redistribute to lists, requires prior specific permission and/or a fee. Request permissions from permissions@acm.org.

PEARC '19, Fuly 28-August 1, 2019, Chicago, IL, USA

(ㄷ) 2019 Copyright held by the owner/author(s). Publication rights licensed to ACM ACM ISBN 978-1-4503-7227-5/19/07 . .\$15.00

https://doi.org/10.1145/3332186.3332255 precision scores than pre-trained CRF models included in ABNER. However, early results from NeuroNER training with on author annotations shows very promising improvement on predicting the important words from the documents.

\section{KEYWORDS}

entity extraction, neural networks, text mining, Domain Informational Vocabulary Extraction (DIVE)

\section{ACM Reference Format:}

Amit Gupta, Weijia Xu, Pankaj Jaiswal, Crispin Taylor, and Jennifer Regala. 2019. Extracting Domain Information using Deep Learning. In Practice and Experience in Advanced Research Computing (PEARC '19), July 28-August 1, 2019, Chicago, IL, USA. ACM, New York, NY, USA, ?? pages. https://doi.org/ 10.1145/3332186.3332255

\section{INTRODUCTION}

The explosion of scientific information, particularly in the form of journal and conference articles is ever present today. Due to ease of maintenance, cross-referencing and delivery to the reader, these articles are now predominantly found in the digital form and even older physical copies of articles are now being converted to digital format. Furthermore, these articles often mark the first appearance of a novel concept, idea or terminology that is accessible to other experts of the field. The need for identification and curation of this information is critical to its accessibility by other relevant experts of the field and forms an important component of ongoing scientific progress. This task however requires significant amounts of human expertise in the relevant domain and due to the scale and scope of the rate of such publications, manual efforts have largely failed to keep pace. Therefore a computational approach to help synthesize this information is needed for this urgent problem.

We have created Domain Informational Vocabulary Extraction (DIVE) to automatically process article text, extract relevant entities and also provide an interface for expert user curation [? ? ]. The system includes a set of tools and web services that can ingest manuscripts to be published for entity extraction. DIVE uses a combination of methods like regular expressions rules, keyword dictionary and checking against known ontolgoies to automatically identify entities. DIVE also has a web interface to allow authors of each paper to validate and select top ten most relevant entities to be included at end of the publication[? ]. We have integrated a live deployment of DIVE into the publication pipeline of two 
prominent journals (Plant Physiology and The Plant Cell) maintained by the American Society of Plant Biologists (ASPB) from March, 2018. Since then, DIVE has processed 486 articles. Through author curation process, we have learned a total of 24,430 entities. In deployment, DIVE is therefore proving to be very instrumental in providing a mechanism to collect author inputs on an ongoing basis thereby helping build a large annotated datasets. On average, DIVE can extract 50 entities per articles. However, one of requirements in practice is to select top 10 most relevant entities to be included at end of a publication. The DIVE workflow currently enables author to carry out a curation step and select top 10 most important entities through its web based interface [?]. While the automatic entities extraction have showed reasonable recall, the precision of automatic entities extraction is relative low comparing to author curation.

To improve the quality of DIVE's extraction results we are exploring deep learning methods that have demonstrated promising performance at various human intelligence tasks [? ? ]. We adapted the NeuroNER tool [?] that trains a deep neural network [? ?] to identify important entities to the article. For reference, we compared results against A Biomedical Named Entities Recognizer (ABNER) which uses conditional random field (CRF) trained on two different biological corpora. However, ABNER doesn't provide rankings or infer importance of recognized entities. Our results show while DIVE's methodology have better performance over ABNER's built in pretrained CRF models, our model trained based on NeuroNER has demonstrated boost in both precision and recall.

In this paper, we first discuss some related work and background of the project in section 2. We then give an introduction of DIVE system in section 3 . We summarize results and statistics collected from first 10 months since it is in service and how we generate a training/validation gold standard dataset based on user curation in section 4 . In section 5 , we describe our testing and results. We discuss results and conclude in section 6 .

\section{RELATED WORK}

In text mining, named entity recognition is a common task for extracting information. The goal of the task is to identify and classify phrases in the corpus to predefined categories, such as, names of persons, organizations, locations, times and dates numerical values, and percentages etc. . Developing systems to automatically extract named entities are motivated through several contest and challenges and become a research topics since early 2000s Named entities recognition requires leveraging linguistics grammar-based models. Early works were centered on handcrafted and rule based algorithms. Rule based systems can exploit features within the specific language to improve the system performance [? ]. Although a rule-based system can be highly efficient for a specific domain, the successes relies on integration of domain knowledge which can be expensive to develop and hard to transfer.

Machine learning techniques have been adopted to learn features automatically in recent years. Supervised learning methods require a curated training data in which named entities have been identified and properly labeled. A typical workflow starts with processing the raw text to generate various features, such as annotations, position, part-of-speech tags. Supervised learning methods are then used to derive an inference model based on training data set. Various learning methods have been explored over the years including, Hidden Markov Model [? ], support vector machine [? ], maximum entropy Markov model (MEMM) based systems [? ], Logistic Expression based systems [?], and Conditional Random Field [? ]. Just recently, deep neural network for named entity recognition are also proposed [?]. NeuroNER uses a deep recurrent neuron network to identify and classify named entities. However, supervised learning methods require considerable amount of training data, which may be difficult to achieve. Unsupervised and semi-supervised learning methods are also proposed ([? ?]). For more details and other approaches, readers can refer to a recent survey [?]. Still, successful solutions are often tightly coupled with the underlying language models and domains. It remains as a challenging problem to port models and algorithms working well for one domain to another problem domain. Substantial amount efforts have focused on algorithm improvements in a well tuned, domain specific, scenario in practice. Thus far, no universal algorithm exists that can work reasonably well across a broad swath of domains.

The flourish of different entity recognition methods have resulted various tools and libraries for entity recognition in practice. Two well-known open source state-of-the-art libraries for general domains are spaCy and Stanford Named Entity Recognizer. The spaCy is a python package provides comprehensive natural language processing support. In the latest version, the implementation also leverages convolutional neuron network to improve parsing and inference ([? ]). Stanford Named Entity Recognizer is a java library and implements linear chain conditional random field sequence mode ([? ]). Both libraries include pre-built general models for English language models and support of training new models with customized labels from training dataset. In addition to these two models, ensemble approaches combining multiple techniques have also been reported for specific use cases in practice. Liu and Zhou proposed a system using Linear CRF and cluster-based approach for recognizing entities from English tweets ([? ]). Ensemble classifiers and rule-based approach have been combined in entity recognition in other languages ([? ? ]. Rule based approach has also been used in conjunction with $\mathrm{CRF}$ techniques for biomedical domains ([?]).

We have described below, the two notable tools that have different methodologies and also have applicability to entity extraction from biological literature. We have therefore chosen to use them to compare against DIVE in the rest of this paper:

\subsection{ABNER}

A Biological Named Entity Recognizer (ABNER) is a tool that automatically detects and tags biological entities like protein and genes in natural language text [? ]. It is implemented in Java and also features a GUI interface where users can manually input sections of text for annotation. It uses Conditional Random Fields [? ] a supervised machine learning algorithm that uses a probabalistic graphical model to enable it to detect and label relevant tokens. It comes packaged with two models, trained on the BioCreative [? ] and NLPBA [? ] corpora. A limitation of this method is that it requires a large amount of human annotated data to train the model and these are relatively small corpora containing short stanzas. 


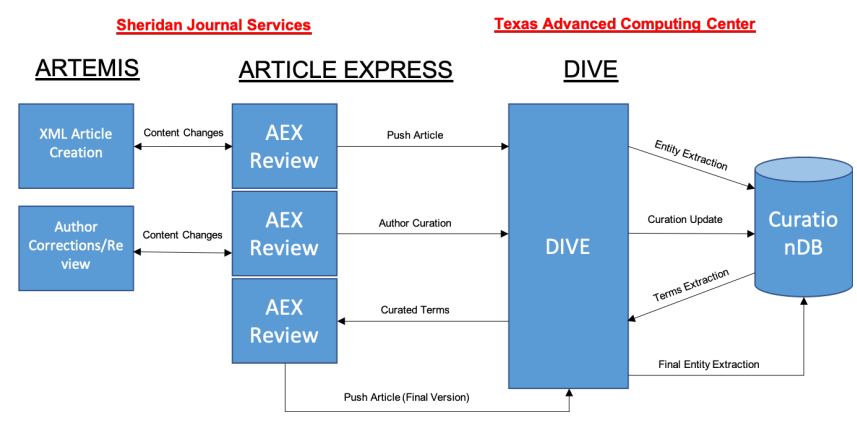

Figure 1: DIVE Integration

\subsection{NeuroNER}

NeuroNER is a named entity recognizer that employs artificial neural networks (specifically a Long Short Term Memory Network [?]) to detect entities in text data [?]. Internally this tool functions as an easy to use wrapper around TensorFlow [? ] to train its network and for running prediction on text data. It also comes with other useful features such as an annotation tool (BRAT) to prepare its datasets. While NeuroNER comes with pretrained models, it allows for training on a user provided test dataset and can thereby be adapted to any corpus. In this paper, we use our own dataset (described later in section ??) to train NeuroNER's built in networks on articles submitted to DIVE for the entity detection task.

\section{DIVE SYSTEM}

Domain Informational Vocabulary Extraction is a system we created to extract entities of interest from corpora of scientific journal articles belonging to a given domain. It uses an ensemble of methods like regular expression rules, keyword dictionaries and domain ontology to extract entities and stores them in its relational back end database . Further architectureal details on its entity detection and extraction process can be found in [? ]. It also features a web interface [?] that is used to expose this data to paper authors, who can then correct already detected entities and even contribute new ones. Author annotations contributed area also stored and tracked in a relational database. This important feedback loop created by DIVE's web interface enables it to be placed in the end to end process of the articles creation. This in turn enables the identification and expert curation of novel entities from the moment of creation of the article.

We have integrated DIVE into the publication pipeline of two prominent journals, namely The Plant Cell and Plant Physiology of the American Society of Plant Biologists (ASPB). The architecture of this integration is shown in Figure ??. We see that DIVE's entity extraction and author curation functionality being a part of all the stages of the article proof creation to its final version. This proof of concept deployment went live in March 2018 and has been extracting entities from articles submitted to these journals since then along with author annotations to the extracted entities. At the time of this writing we have collected and curated 486 full length articles. For reference a monthly distribution of articles submitted can be seen in Figure ??.

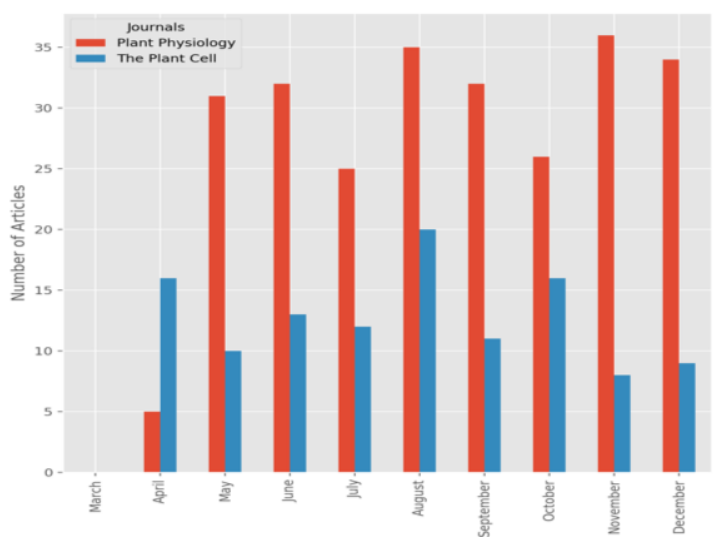

Figure 2: Articles Submitted Montly Distribution

\section{RESULTS}

\subsection{Gold Standard Dataset Creation}

We first describe the data that we use as the gold standard to compare performance between the various entity extraction tools. One of DIVE's components is a web interface that allows authors to curate entities detected in their paper. When using the web interface, the authors are initially shown the top ten entities that were found in their paper. The author may also choose to add more entities and perform curation edits/deletes on the existing ones. Once the authors have concluded their curation activity, we have a list of entities most relevant to the paper that have been essentially reviewed, correct and/or verified by the author (who is the expert on the technical content of the paper). All of this information is stored in the DIVE's backend relational database and can be queried. This thus enables us to collate this information and along with the actual article text forms our gold standard dataset for entity extraction task for this corpus. At present, the gold standard dataset used in this paper comprises of 443 articles that DIVE had curated (beginning in January 2018) and 8787 author curated entities ${ }^{1}$. We also present in Table (??) the statistical summary of curated entities per article. This dataset is being built based on actual article submissions to ASPB journals. While there may be implicit weighting of terms due to relative numbers of different types of articles (for example methodology papers, position/literature review papers and novel research papers etc), for the purposes of this paper we treat each article type equally and ignore any such implicit weighting effects on the curated terms. With more time in deployment and increased author participation, we expect this dataset to grow and help train and enable more sophisticated models for entity extraction.

\footnotetext{
${ }^{1}$ Our initial deployment displayed all entities in the article. However, after a few months in deployment and with author feedback, we modified this to display the top ten as authors felt this would be sufficient to capture the most important entities of their papers. However we still give the authors the option of adding more entities manually as they see fit during their curation. Since our data is cumulative of our deployment period, this results in the number of curated entities not being a direct multiple of the number of articles (i.e number of articles x 10).
} 
Table 1: Statistical Summary of Entities curated per article

\begin{tabular}{cc}
\hline Measure & Entity curated per article \\
\hline Mean & 16.015801 \\
Std Deviation & 10.759074 \\
Minimum & 2.000000 \\
$25 \%$ & 9.000000 \\
$50 \%$ & 12.000000 \\
$75 \%$ & 20.000000 \\
Maximum & 127.000000 \\
\hline
\end{tabular}

\subsection{NeuroNER Training: Dataset and Hardware}

NeuroNER input data is provided in the BRAT standoff format [?]. This format consists of individual text files that contain full article text and are accompanied by their respective annotation files which provide the start and end index numbers for each occurrence of every entity present in the article text along with their labels. We generated this input data for our entire corpus of 443 articles submitted to DIVE. We also organized the units of text in our data in 3 ways:

- Full article text: Here each text file consists of the entire text from the article. Its accompanying annotation file points to the location of entities confirmed by the author in the article text. It does so for all occurrences of each entity that is present in the text file. Therefore the unit of text being used for training in this dataset is an entire articles text.

- 300 word buckets: Here each text file consists of a chunk of 300 words from an article. The accompanying annotation file points to the location of entities confirmed by the author that is present in that chunk of text only. For each DIVE article, its entire text is broken up into smaller text files of 300 contiguous word chunks and the accompanying annotation file is generated. Therefore the unit of text being used for training in this dataset is a 300 word excerpt from an article.

- $\mathbf{1 0 0 0}$ word buckets: Here each text file consists of a chunk of 1000 words from an article. The accompanying annotation file points to the location of entities confirmed by the author that is present in that chunk of text only. For each DIVE article, its entire text is broken up into smaller text files of 1000 contiguous word chunks and the accompanying annotation file is generated. Therefore the unit of text being used for training in this dataset is a 1000 word excerpt from an article.

We created 2 labels for annotation in these datasets using the author annotations obtained from DIVE's database. Proteins and Genes formed the bulk of entities of interest to the authors curating articles in DIVE. Proteins and Genes verified and curated by authors were marked as "Positive Author Interest" indicating that these are the types of entities we would like the algorithm to learn. Often there are numerous other entities present and detected by DIVE (for example, a chemical reagent name or an apparatus name) in an article, that the authors dont consider topically defining for their paper. We therefore marked all other entities (besides Proteins and Genes) as "Negative Author Interest" indicating that these are the types of entities we would like the algorithm to learn so that they may be filtered out at a later stage by DIVE. These two labels are used for annotation in our dataset for NeuroNER.

Furthermore, for optimal training, we split the input data into Training (80\%), Validation (10\%) and Testing (10\%) sets. Due to computational expense of the training process (shown in Section (??), $\mathrm{k}$-fold cross validation was not done in our experiments. The exact number of articles, units of text and dataset splits for each of these datasets is shown in Table (??). NeuroNER training was conducted on Texas Advanced Computing Center's Maverick2 system [? ] using a Intel(R) Xeon(R) E5-2620 v4 CPU and single NVidia 1080-TI GPU. For these prelimiary experiments, only single node was used for this training.

\subsection{Performance and Discussion}

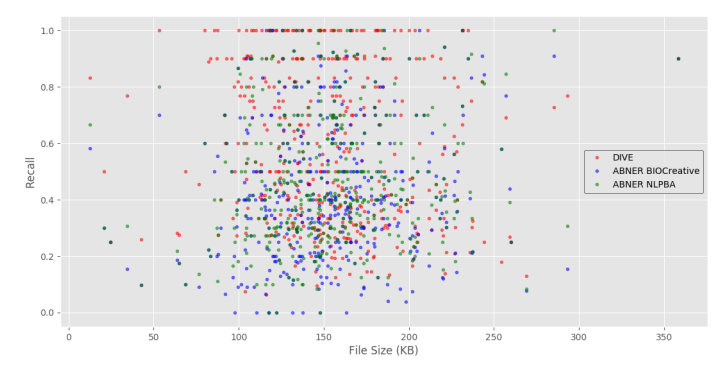

Figure 3: Recall Comparison Results vs Article Filesize

Figure ?? shows the precision and recall performance for the comparisons between DIVE, ABNER BioCreative, ABNER NLPBA and NeuroNER trained on DIVE's curated corpus. For DIVE, ABNER BioCreative and ABNER NLPBA models, we used the full article dataset. For NeuroNER, we used the 3 variants of the dataset with different sized units of text as described above. We initially see in this figure that recall perfomance of DIVE is higher than both builtin ABNER models. Furthermore, A DIVE's precision performance is about $7 \mathbf{x}$ times of ABNER models. ABNER models dont provide any ranking and return a very large number of entities which help their recall numbers but significantly hurt their precision. Table ?? shows the statistical summary of the Recall numbers over all articles in the Gold Standard dataset. As can be seen, DIVE has better averages and across all quantiles of this data. While DIVE has better average performance than both ABNER models, it does have higher variance within its results. Figure ?? shows the recall results across all the articles of the dataset, sorted by filesize. The high variance in both DIVE and ABNER results is also evident. Furthermore, DIVE and ABNER's models correspond to the class label of "Positive Author Interest" used for NeuroNER models. Neither DIVE nor ABNERs models have a mechanism to detect candidates for elimination.

With respect to NeuroNER, we see that they significantly outperform both DIVE (by more than a factor of $2 \mathbf{x}$ ) and ABNERs models for precision on all 3 variants of the dataset. We also further see the effect of segmentation of input text on NeuroNER's precision numbers where smaller units of text improve the precision of NeuroNER prediction. At peak performance, NeuroNER's precision tops at about $80 \%$. We see similar trends in the recall numbers for 
Table 2: NeuroNER Training Dataset

\begin{tabular}{lccccc}
\hline Dataset & Total Articles & $\begin{array}{c}\text { Total Buckets } \\
\text { (i.e units of text) }\end{array}$ & Training & Validation & Testing \\
\hline 300 Word Buckets & 443 & 17691 & 372 & 36 & 35 \\
1000 Word Buckets & 443 & 5466 & 349 & 40 & 54 \\
Full Article Text & 443 & 443 & 345 & 51 & 47 \\
\hline
\end{tabular}

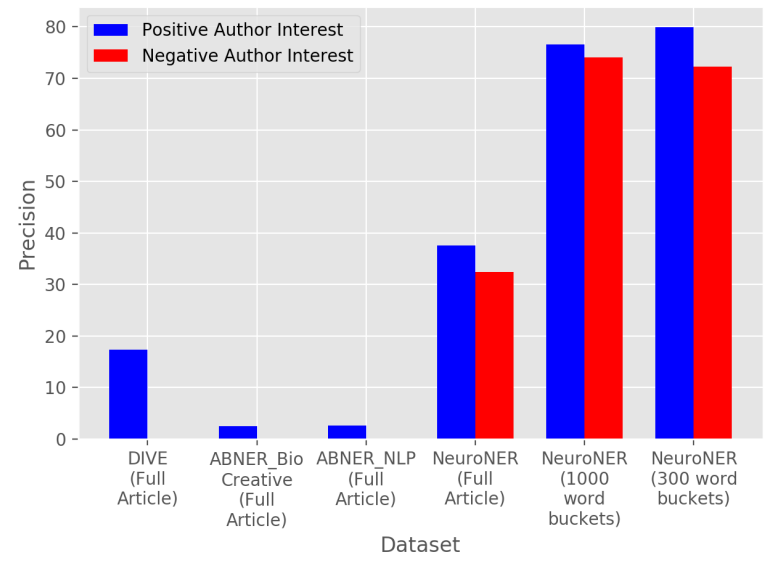

(a) Precision across Datasets/Systems

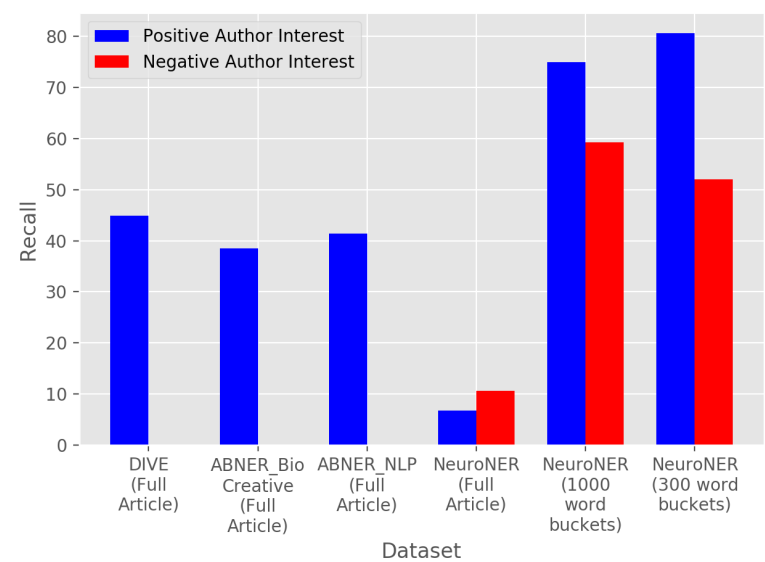

(b) Recall across Datasets/Systems

Figure 4: Precision and Recall across Datasets and Systems

Table 3: Statistical Summary

\begin{tabular}{lccccccc}
\hline System & Min & Max & Mean & 25\%-ile & $50 \%$-ile & 75\%-ile & Standard Deviation \\
\hline DIVE & 0.062500 & 1.000000 & 0.582544 & 0.333333 & 0.529412 & 0.833333 & 0.275227 \\
ABNER BIOCreative & 0.000000 & 1.000000 & 0.434602 & 0.250000 & 0.400000 & 0.600000 & 0.240368 \\
ABNER NLP & 0.000000 & 1.000000 & 0.468504 & 0.300000 & 0.416667 & 0.600000 & 0.228402 \\
\hline
\end{tabular}

NeuroNER except when we use larger units of text corresponding to the full article text. Due to limitations in computation time, the training for NeuroNER results included in this paper were limited to 4 epochs. We feel this could be a possible explanation for this and we would like to investigate this anomaly further in future extension of this study.

Figure ?? shows us a deeper look into the characteristics of text segmentation on NeuroNER as it proceeds through the training epochs. Shown here are the precision and recall performance for both annotation labels across the training epochs. We see again here that NeuroNER performance for precision and recall is far superior on smaller units of text.

Figure ?? also presents some insight into the effects of text segmentation on the training time required to train the NeuroNER model. We see here that smaller segmentation of input text, marginally increases the training time. We also notice that NeuroNER training on CPU outperforms that of GPU, thus leading us to believe that there may be room for significant optimization/parallelization of its internal computations. We would like to investigate both these issues deeper in future work and help reduce its training time for this application.

\section{CONCLUSION AND FUTURE WORK}

We presented an exploration of using deep neural network for recognizing important terms from biological journal articles. For this study, we leveraged curated results from DIVE system which is in live production for American Society for Plant Biologists. We present details on how we can generate a growing Gold Standard entity dataset from the corpus being maintained. While DIVE's comparatively superior performance on this dataset against previous systems with similar goals like the two CRF based ABNER models, the importance prediction can be further enhanced by using deep learning methods. We adapted NeuroNER for this purpose. We are working on to integrate the deep learning model with existing DIVE system for a system capable of automated progressive improvement. As can be inferred from Figure ??, the time to train an 


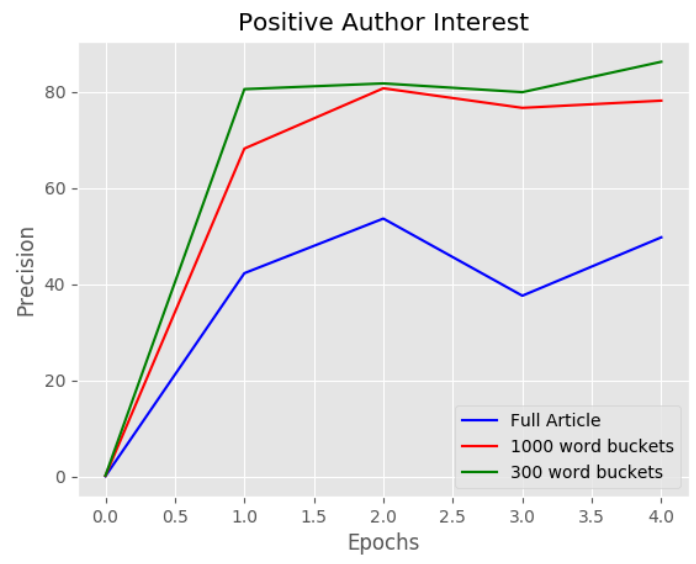

(a) Precision for Positive Author Interest

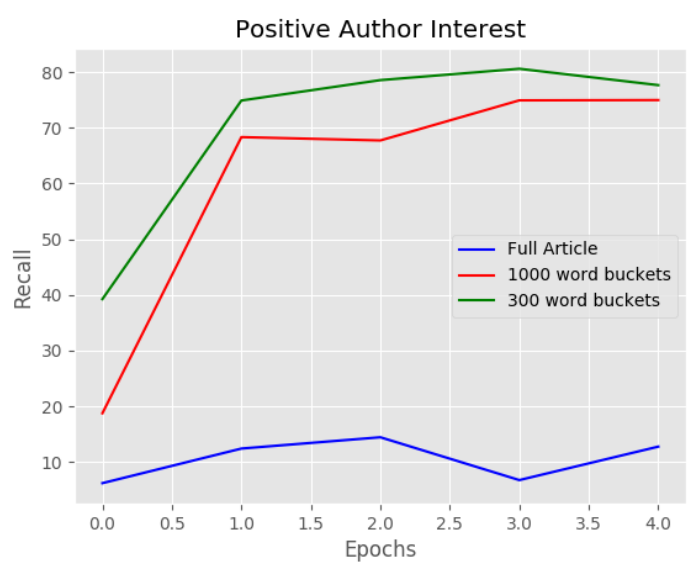

(c) Recall for Positive Author Interest

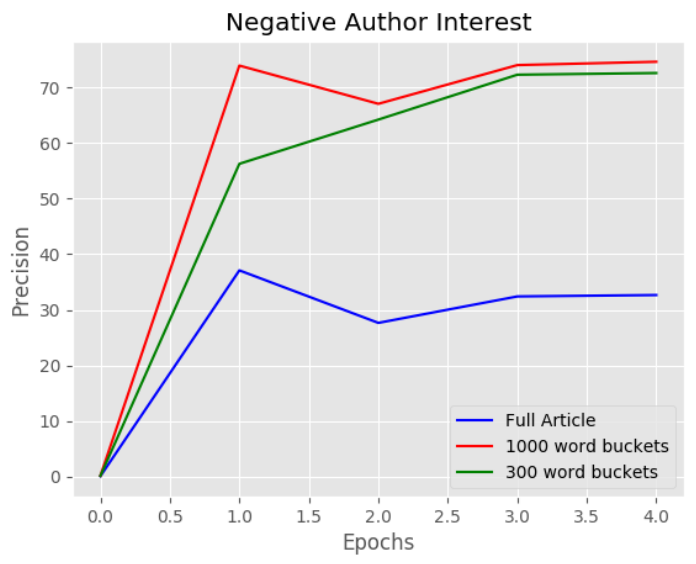

(b) Precision for Negative Author Interest

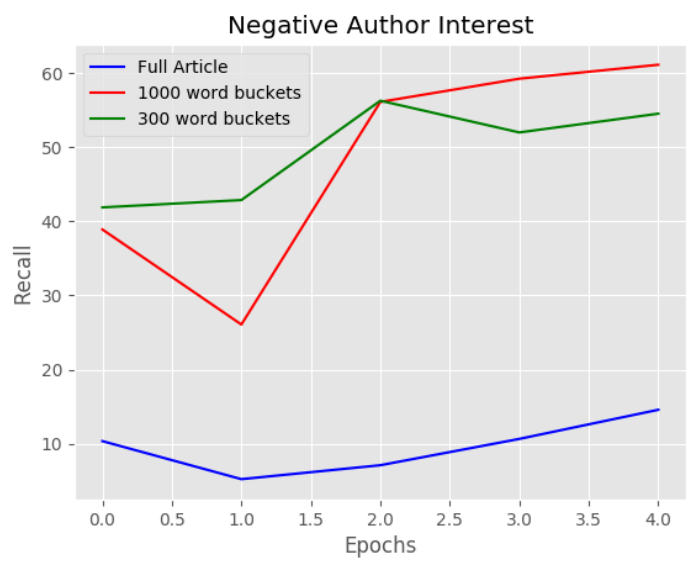

(d) Recall for Negative Author Interest

Figure 5: Precision and Recall for Positive and Negative Author Interest Labels across training epochs

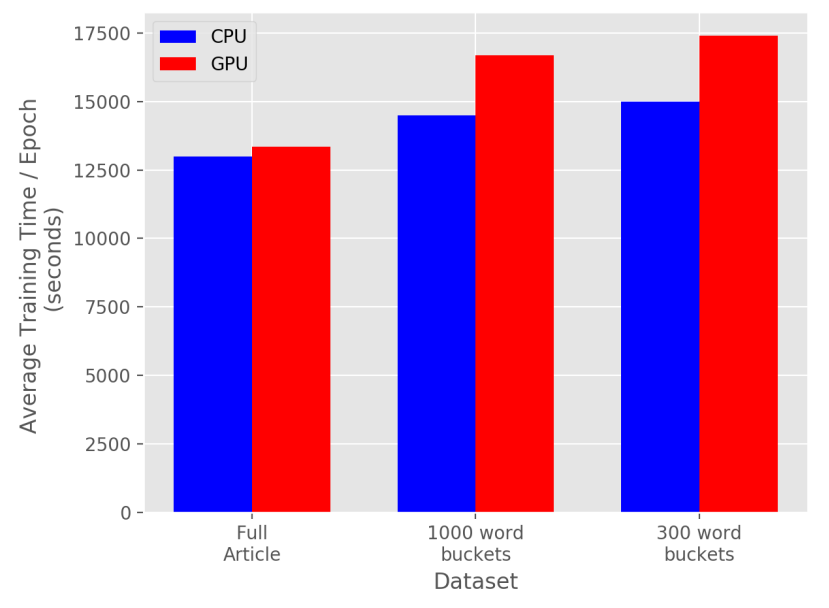

Figure 6: Average Training Time Per Epoch effective neural network for this application scenario is non-trivial. We would therefore also like to investigate opportunities to reduce this training time by strategic distribution of data and computation loads. We are also investigating other areas of performance optimization such as bucket size of split and other parameters to further improve its performance and running time. Reduction in training time will enable us to train a model for more iterations and potentially better accuracy. Successful integration of this technique in DIVE's live deployment for ASPB journal submission system will also provide us with the unique opportunity to gather author feedback on the models accuracy in identifying the most significant entities in their articles and comparison against human expert performance for this task. Overall, our approach is extensible and its has a modular design. Therefore the approach proposed here can be applied to train models for technical documents from other domains. 


\section{ACKNOWLEDGMENTS}

This research is partially supported by CyVerse (NSF award DBI0735191 and DBI-1265383) and the Gramene, a Comparative Plant Genomics Database (NSF award IOS-1127112). 